\title{
Global Optimal Solutions to General Sensor Network Localization Problem
}

\author{
N. Ruan ${ }^{\mathrm{a}}$, D.Y. Gao ${ }^{\mathrm{a}, \mathrm{b}, *}$ \\ ${ }^{a}$ School of Sciences, Information Technology and Engineering, \\ Federation University Australia, Mt Helen, VIC 3353, Australia \\ ${ }^{b}$ Research School of Engineering, Australian National University, Canberra, ACT 0200, \\ Australia
}

\begin{abstract}
Sensor network localization problem is to determine the position of the sensor nodes in a network given pairwise distance measurements. Such problem can be formulated as a polynomial minimization via the least squares method. This paper presents a canonical duality theory for solving this challenging problem. It is shown that the nonconvex minimization problem can be reformulated as a concave maximization dual problem over a convex set in a symmetrical matrix space, and hence can be solved efficiently by combining a general (linear or quadratic) perturbation technique with existing optimization techniques. Applications are illustrated by solving some relatively large-scale problems. Our results show that the general sensor network localization problem is not NP-hard unless its canonical dual problem has no solution. Fundamental ideas for solving general NP-hard problems are discussed.
\end{abstract}

Keywords: Sensor network localization; Canonical duality theory; Perturbation method; Global optimization; NP-Hard problems

\section{Introduction}

Sensor network localization is an important problem in communication and information theory, and has attracted an increasing attention [3, ㅇ, 26,

\footnotetext{
${ }^{*}$ Corresponding author

Email addresses: n.ruan@ballarat.edu.au (N. Ruan), d.gao@ballarat.edu.au, david.gao@anu.edu.au (D.Y. Gao) 
35, 45]. The information collected through a sensor network can be interpreted and relayed far more effectively if it is known where the information is coming from and where it needs to be sent. Therefore, it is often very useful to know the positions of the sensor nodes in a network. Wireless sensor network consists of a large number of wireless sensors located in a geographical area with the ability to communicate with their neighbors within a limited radio range. Sensors collect the local environmental information, such as temperature or humidity, and can communicate with each other. Wireless sensor network is applicable to a range of monitoring applications in civil and military scenarios, such as geographical monitoring, smart homes, industrial control and traffic monitoring. There is an urgent need to develop robust and efficient algorithms that can identify sensor positions in a network by using only the measurements of the mutual distances of the wireless sensors from their neighbors, which is called neighboring distance measurements. The advance of wireless communication technology has made the sensor network a low-cost and highly efficient method for environmental observations.

Sensor network localization can also be formulated as an optimization problem by least squares method. However, this problem is nonconvex with many local minimizers. To find global optimal solutions by traditional theories and local-search methods is fundamentally difficult. It turns out that the general sensor localization problem has been considered to be NP-hard [2, 32]. Several approximation methods have been developed for solving this difficult optimization problem (see [33] and references cited therein). The semi-definite programming (SDP) and second-order cone programming (SOCP) relaxations are two of the most popular methods studied recently [4, 34, 41, 43]. The basic idea of SDP relaxation is to think of the quadratic terms as new variables subject to a linear matrix inequality. The SOCP relaxation is developed in a similar way. For both SDP and SOCP relaxation, computed sensor locations are not accurate when the solution of the localization problem is not unique. This is because many numerical schemes, such as primal-dual and interior point methods, for SDP or SOCP relaxation often return to the analytic centre of the solution set. These solutions are, in general, not global optimal solutions.

Mathematically speaking, the localization problem in $\mathbb{R}^{d}$ can be stated as follows [1, 7]: Consider a sensor network in $\mathbb{R}^{d}$ with $m$ anchors and $n$ sensors. An anchor is a node whose location $a_{k} \in \mathbb{R}^{d}$, where $k=1, \cdots, m$, is known, and a sensor is a node whose location $x_{i} \in \mathbb{R}^{d}$, where $i=1, \cdots, n$, is yet to be determined. For a pair of sensors $x_{i}$ and $x_{j}$, their Euclidean 
distance is denoted as $d_{i j}$. Similarly, for a pair of sensor $x_{i}$ and anchor $a_{k}$, their Euclidean distance is denoted as $e_{i k}$. In general, not all pairs of sensor /sensor and sensor/anchor are known, so the known pair-wise distances of sensor/sensor and sensor/anchor are denoted as $(i, j) \in \mathcal{A}_{d}$ and $(i, k) \in \mathcal{A}_{e}$, respectively. However, if we directly apply the general least squares method, the computation is very expensive and not practical for large problems [25].

Canonical duality theory developed from nonconvex analysis and global optimization (see [13, 23]) is a powerful methodology, which has been used successfully for solving a large class of challenging problems in various disci-

plines. See, for example, [17, 20, 21, 28, 44]. This paper presents an effective perturbation method based on the canonical duality theory to solve the general sensor network localization problem. Our main contribution is to show that this nonconvex optimization problem is not NP-hard unless its canonical dual problem has no solution. The rest of this paper is organized as follows. In the next section, we first reformulate the original problem as an optimization problem, where the decision variable is expressed in tensor (matrix) forms. In Section 3, the canonical duality theory is discussed in matrix space and a general analytical solution form is obtained by a complementary-dual principle. In section 4, the general sensor localization problem is first reformulated in vector space and then transformed as a concave maximization dual problem over a convex feasible space $\mathcal{S}_{a}^{+}$. Based on the triality theory, a quadratic perturbation method is proposed, which shows that the nonconvex sensor network optimization problem is not NP-hard unless its canonical dual problem has no solution in $\mathcal{S}_{a}^{+}$. Section 5 presents some concrete numerical experiments for sensor localization problems with two, 18, 20 and 200 sensors. The cases with noise are also considered. Results are compared with standard semi-definite programming method. Concluding remarks are given in the last section.

The notations used in this paper are: $\mathbb{R}$ denotes the set of real numbers; $A^{T}$ denotes the transpose of matrix $A$. For a finite set $S,|S|$ denotes its cardinality and the bilinear form $\left\langle u, u^{*}\right\rangle$ is simply the scalar product of two vectors or tensors.

\section{Problem Statement}

Let us consider a general sensor network localization problem, where the sensor locations are to be determined by solving the system of nonlinear 
equations

$$
\begin{aligned}
\left(\mathcal{P}_{0}\right): \quad\left\|\mathbf{x}_{i}-\mathbf{x}_{j}\right\| & =d_{i j}, \quad(i, j) \in \mathcal{A}_{d}, \\
\left\|\mathbf{x}_{i}-\mathbf{a}_{k}\right\| & =e_{i k}, \quad(i, k) \in \mathcal{A}_{e} .
\end{aligned}
$$

Here, the vectors $\mathbf{a}_{k}, k=1, \cdots, m$, are specified anchors, where

$$
\left\|\mathbf{x}_{i}-\mathbf{x}_{j}\right\|=\sqrt{\sum_{\alpha=1}^{d}\left(x_{i}^{\alpha}-x_{j}^{\alpha}\right)^{2}}
$$

denotes the Euclidian distance between locations $\mathbf{x}_{i}$ and $\mathbf{x}_{j} \in \mathbb{R}^{d}, i=$ $1, \cdots, n ; j=1, \cdots, n$, and

$\mathcal{A}_{d}=\left\{(i, j) \in[n] \times[n] \mid\left\|\mathbf{x}_{i}-\mathbf{x}_{j}\right\|=d_{i j}, \quad i<j, \quad d_{i j}\right.$ are given distances $\}$, $\mathcal{A}_{e}=\left\{(i, k) \in[n] \times[m] \mid\left\|\mathbf{x}_{i}-\mathbf{a}_{k}\right\|=e_{i k}, \quad e_{i k}\right.$ are given distances $\}$,

where $[N]=\{1, \cdots, N\}$ for any integer $N$.

For a small number of sensors, it might be possible to compute sensor locations by solving equations (11)-(2). However, solving this algebraic system can be very expensive computationally when the number of sensors is large.

By the least squares method [37], the sensor network localization problem $\left(\mathcal{P}_{0}\right)$ can be reformulated as a fourth-order polynomial optimization problem stated below.

$$
\begin{aligned}
\left(\mathcal{P}_{1}\right): \min \{\Pi(\mathbf{X}) & =\sum_{(i, j) \in \mathcal{A}_{d}} \frac{1}{2} w_{i j}\left(\left\|\mathbf{x}_{i}-\mathbf{x}_{j}\right\|^{2}-d_{i j}^{2}\right)^{2} \\
& \left.+\sum_{(i, k) \in \mathcal{A}_{e}} \frac{1}{2} q_{i k}\left(\left\|\mathbf{x}_{i}-\mathbf{a}_{k}\right\|^{2}-e_{i k}^{2}\right)^{2}\right\},
\end{aligned}
$$

where $\mathbf{X}=\left[x_{1}, x_{2}, \cdots, x_{n}\right]=\left\{x_{i}^{\alpha}\right\} \in \mathbb{R}^{d \times n}$ is a matrix with each column $x_{i}$ being a position in $\mathbb{R}^{d}, w_{i j}, q_{i k}>0$ are given weights. Obviously, $\mathbf{X}$ are true sensor locations if and only if the optimal value is zero. This nonconvex optimization problem appears extensively in mathematical physics 24], chaotic dynamics [36], numerical algebra [37], computational biology [44], as well as finite element analysis of structural mechanics [5, 38]. Due to the nonconvexity, this problem could have many local minimizers. It is fundamentally 
difficult, or even impossible, to find global optimal solutions by traditional direct methods. In the following, we shall see that by using the canonical duality theory, this nonconvex minimization problem can be reformulated as a concave maximization dual problem over a convex set under certain conditions, which can be solved efficiently by a proposed perturbation method.

\section{Canonical duality theory: A brief review}

The canonical duality theory is composed mainly of 1) a canonical transformation; 2) a complementary-dual principle; 3) a triality theory. This theory can be demonstrated by solving the following general nonconvex problem (the primal problem $(\mathcal{P})$ in short)

$$
(\mathcal{P}): \min _{\mathbf{x} \in \mathcal{X}_{a}}\left\{\Pi(\mathbf{x})=\frac{1}{2}\langle\mathbf{x}, \mathbf{A} \mathbf{x}\rangle-\langle\mathbf{x}, \mathbf{f}\rangle+W(\mathbf{B} \mathbf{x})\right\},
$$

where $\mathcal{X}_{a} \subset \mathbb{R}^{d \times n}$ is a given feasible space, $\left\langle\mathbf{x}, \mathbf{x}^{*}\right\rangle$ denotes the bilinear form between $\mathbf{x}$ and its dual variable $\mathbf{x}^{*}, \mathbf{f} \in \mathcal{X}_{a}^{*} \subset \mathbb{R}^{n \times d}$ is a given matrix, $\mathbf{A}: \mathcal{X}_{a} \rightarrow \mathcal{X}_{a}^{*}$ is a given self-adjoint linear operator, $\mathbf{B}$ is a linear operator which assign each $\mathbf{x} \in \mathcal{X}_{a}$ to a (deformation gradient-like) variable in a linear space $\mathcal{W}_{a}$, on which, $W(\mathbf{w}): \mathcal{W}_{a} \rightarrow \mathbb{R}$ is a well-defined differentiable nonconvex function.

The canonical transformation is to choose a "geometrically admissible" nonlinear operator (see [13])

$$
\boldsymbol{\xi}=\Lambda(\mathbf{x}): \mathcal{X}_{a} \rightarrow \mathcal{E}_{a}
$$

which maps the convex set $\mathcal{X}_{a}$ into a convex set $\mathcal{E}_{a}$, and a canonical function $V: \mathcal{E}_{a} \rightarrow \mathbb{R}$ such that the nonconvex functional $W(\mathbf{w})$ can be recast in a canonical form $W(\mathbf{B x})=V(\Lambda(\mathbf{x}))$. Thus, the primal problem $(\mathcal{P})$ can be written in the following canonical form:

$$
(\mathcal{P}): \min _{\mathbf{x} \in \mathcal{X}_{a}}\{\Pi(\mathbf{x})=V(\Lambda(\mathbf{x}))-U(\mathbf{x})\}
$$

where $U(\mathbf{x})=\langle\mathbf{x}, \mathbf{f}\rangle-\frac{1}{2}\langle\mathbf{x}, \mathbf{A} \mathbf{x}\rangle$. By the definitions introduced in [13], a nonlinear operator $\Lambda(\mathbf{x}): \mathcal{X}_{a} \rightarrow \mathcal{E}_{a}$ is said to be geometrically admissible if it can be used as a (deformation) measure such that the canonical transformation $W(\mathbf{B x})=V(\Lambda(\mathbf{x}))$ satisfies certain necessary (geometrical and physical) conditions, for examples, the objectivity and isotropy. Let

$$
\mathcal{R}=\left\{\mathbf{R} \in \mathbb{R}^{m \times m} \mid \mathbf{R}^{T}=\mathbf{R}^{-1}, \operatorname{det} \mathbf{R}=1\right\}
$$


be a proper orthogonal rotation group in $\mathbb{R}^{m}$.

Definition 1 (Objectivity and Isotropy [13] ). A subset $\mathcal{W}_{a}$ is said to be objective if

$$
\mathbf{R w} \in \mathcal{W}_{a} \forall \mathbf{w} \in \mathcal{W}_{a} \text { and } \forall \mathbf{R} \in \mathcal{R} \text {. }
$$

A real-valued function $W: \mathcal{W}_{a} \rightarrow \mathbb{R}$ is said to be objective if its domain is objective and

$$
W(\mathbf{R w})=W(\mathbf{w}) \forall \mathbf{w} \in \mathcal{W}_{a} \text { and } \forall \mathbf{R} \in \mathcal{R}
$$

A subset $\mathcal{W}_{a}$ is said to be isotropic if

$$
\mathbf{w} \mathbf{R} \in \mathcal{W}_{a} \forall \mathbf{w} \in \mathcal{W}_{a} \text { and } \forall \mathbf{R} \in \mathcal{R}
$$

A real-valued function $W: \mathcal{W}_{a} \rightarrow \mathbb{R}$ is said to be isotropic if its domain is isotropic and

$$
W(\mathbf{w} \mathbf{R})=W(\mathbf{w}) \quad \forall \mathbf{w} \in \mathcal{W}_{a} \text { and } \forall \mathbf{R} \in \mathcal{R} .
$$

Geometrically speaking, the objectivity means that the function $W(\mathbf{w})$ does not depend on rotation, but only on certain objective measure of its variable $\mathbf{w}$. Therefore, the most simple objective function is the right CauchyGreen deformation tensor $\mathbf{C}=\mathbf{w}^{T} \mathbf{w}$ since

$$
\mathbf{C}(\mathbf{R} \mathbf{w})=\mathbf{w}^{T} \mathbf{R}^{T} \mathbf{R} \mathbf{w}=\mathbf{w}^{T} \mathbf{w}=\mathbf{C}(\mathbf{w}) \succeq 0 \quad \forall \mathbf{R} \in \mathcal{R} .
$$

While the isotropy implies that the function $W(\mathbf{w})$ possesses a certain symmetry. Clearly, the left Cauchy-Green deformation tensor $\mathbf{w} \mathbf{w}^{T}$ is an isotropic measure due to the fact

$$
\left(\mathbf{w} \mathbf{R}^{T}\right)\left(\mathbf{w} \mathbf{R}^{T}\right)^{T}=\mathbf{w} \mathbf{w}^{T} \succeq 0 \quad \forall \mathbf{R} \in \mathcal{R} .
$$

The concepts of objectivity and isotropy play important role in Semi-Definite Programming (SDP) and integer programming [16, 19]. Particularly, if $\mathbf{w}$ is a vector, the objectivity is identical to isotropy. Furthermore, if the objective function $W(\mathbf{w})$ is considered as a kinetic energy and $U(\mathbf{x})$ is viewed as the potential energy, then the function $\Pi(\mathbf{x})$ is the original Lagrangian in mathematical physics [13].

The objectivity in science is also refereed as frame invariance, which lays a foundation for mathematical physics and systems theory. In fact, 
the canonical duality theory was originally developed from this concept [13], which is the reason why this theory can be applied not only for modeling and analysis of complex systems, but also for solving a large class of nonconvex/nonsmooth/discrete problems in both mathematical physics and global optimization (see review article [21]).

A differentiable function $V(\boldsymbol{\xi})$ is said to be a canonical function on its domain $\mathcal{E}_{a}$ if the duality mapping $\boldsymbol{\varsigma}=\nabla V(\boldsymbol{\xi})$ from $\mathcal{E}_{a}$ to its range $\mathcal{E}_{a}^{*}$ is invertible. Let $\langle\boldsymbol{\xi} ; \boldsymbol{\varsigma}\rangle$ denote the bilinear form on $\mathcal{E}_{a} \times \mathcal{E}_{a}^{*}$. Thus, for the given canonical function $V(\boldsymbol{\xi})$, its Legendre conjugate $V^{*}(\boldsymbol{\varsigma})$ can be defined uniquely by the Legendre transformation

$$
V^{*}(\boldsymbol{\varsigma})=\operatorname{sta}\left\{\langle\boldsymbol{\xi} ; \boldsymbol{\varsigma}\rangle-V(\boldsymbol{\xi}) \mid \boldsymbol{\xi} \in \mathcal{E}_{a}\right\},
$$

where the notation $\operatorname{sta}\left\{g(\boldsymbol{\xi}) \mid \boldsymbol{\xi} \in \mathcal{E}_{a}\right\}$ stands for finding stationary point of $g(\boldsymbol{\xi})$ on $\mathcal{E}_{a}$. It is easy to prove that the following canonical duality relations hold on $\mathcal{E}_{a} \times \mathcal{E}_{a}^{*}$ :

$$
\boldsymbol{\varsigma}=\nabla V(\boldsymbol{\xi}) \Leftrightarrow \boldsymbol{\xi}=\nabla V^{*}(\boldsymbol{\varsigma}) \Leftrightarrow V(\boldsymbol{\xi})+V^{*}(\boldsymbol{\varsigma})=\langle\boldsymbol{\xi} ; \boldsymbol{\varsigma}\rangle .
$$

By this one-to-one canonical duality, the nonconvex term $W(\mathbf{D x})=V(\Lambda(\mathbf{x}))$ in the problem $(\mathcal{P})$ can be replaced by $\langle\Lambda(\mathbf{x}) ; \boldsymbol{\varsigma}\rangle-V^{*}(\boldsymbol{\varsigma})$ such that the nonconvex function $\Pi(\mathbf{x})$ is reformulated as the so-called Gao and Strang total complementary function [13]:

$$
\Xi(\mathbf{x}, \boldsymbol{\varsigma})=\langle\Lambda(\mathbf{x}) ; \boldsymbol{\varsigma}\rangle-V^{*}(\boldsymbol{\varsigma})-U(\mathbf{x})
$$

By using this total complementary function, the canonical dual function $\Pi^{d}(\boldsymbol{\varsigma})$ can be obtained as

$$
\begin{aligned}
\Pi^{d}(\boldsymbol{\varsigma}) & =\operatorname{sta}\left\{\Xi(\mathbf{x}, \boldsymbol{\varsigma}) \mid \mathbf{x} \in \mathcal{X}_{a}\right\} \\
& =U^{\Lambda}(\boldsymbol{\varsigma})-V^{*}(\boldsymbol{\varsigma}),
\end{aligned}
$$

where $U^{\Lambda}(\boldsymbol{\varsigma})$ is defined by

$$
U^{\Lambda}(\boldsymbol{\varsigma})=\operatorname{sta}\left\{\langle\Lambda(\mathbf{x}) ; \boldsymbol{\varsigma}\rangle-U(\mathbf{x}) \mid \mathbf{x} \in \mathcal{X}_{a}\right\} .
$$

In many applications, the geometrically nonlinear operator $\Lambda(\mathbf{x})$ is usually a tensor-valued quadratic function

$$
\Lambda(\mathbf{x})=\left\{\frac{1}{2}\left\langle\mathbf{x}, \mathbf{H}_{k l} \mathbf{x}\right\rangle\right\}: \mathbb{R}^{d \times n} \rightarrow \mathbb{R}^{n \times n},
$$


where $\mathbf{H}_{k l}: \mathcal{X}_{a} \rightarrow \mathcal{X}_{a}^{*} \forall k, l=1, \ldots, n$ is a symmetrical linear operator. In this case, the canonical dual variable $\varsigma \in \mathcal{E}_{a}^{*} \subset \mathbb{R}^{n \times n}$ is a symmetrical tensor and the total complementary function $\Xi$ can be written in the following form

$$
\Xi(\mathbf{x}, \boldsymbol{\varsigma})=\frac{1}{2}\langle\mathbf{x}, \mathbf{G}(\boldsymbol{\varsigma}) \mathbf{x}\rangle-V^{*}(\boldsymbol{\varsigma})-\langle\mathbf{x}, \mathbf{f}\rangle,
$$

where

$$
\mathbf{G}(\boldsymbol{\varsigma})=\mathbf{A}+\sum_{k, l} \varsigma_{k l} \mathbf{H}_{k l} .
$$

For any given $\varsigma \in \mathcal{E}_{a}^{*}$, the criticality condition $\nabla_{\mathbf{x}} \Xi(\mathbf{x}, \boldsymbol{\varsigma})=0$ leads to the canonical equilibrium equation $\mathbf{G}(\boldsymbol{\varsigma}) \mathbf{x}=\mathbf{f}$. Let

$$
\mathcal{S}_{a}=\left\{\boldsymbol{\varsigma} \in \mathcal{E}_{a}^{*} \mid \operatorname{det} \mathbf{G}(\boldsymbol{\varsigma}) \neq 0\right\} .
$$

Then on $\mathcal{S}_{a}$, the solution to canonical equilibrium equation can be written as $\mathbf{x}=\mathbf{G}^{-1}(\boldsymbol{\varsigma}) \mathbf{f}$. Therefore, replacing the primal variable $\mathbf{x}$ by this generalized solution in $\Xi$, the canonical dual function (14) can be explicitly formulated in the form of

$$
\Pi^{d}(\boldsymbol{\varsigma})=-\frac{1}{2}\left\langle\mathbf{f}, \mathbf{G}^{-1}(\boldsymbol{\varsigma}) \mathbf{f}\right\rangle-V^{*}(\boldsymbol{\varsigma}) .
$$

Theorem 1 (Complementary-Dual Principle [13]). The function $\Pi^{d}(\varsigma)$ is canonically dual to $\Pi(\mathbf{x})$ in the sense that if $\overline{\boldsymbol{s}}$ is a critical point of $\Pi^{d}(\boldsymbol{\varsigma})$, then

$$
\overline{\mathbf{x}}=\mathbf{G}^{-1}(\overline{\boldsymbol{\zeta}}) \mathbf{f}
$$

is a critical point of $\Pi(\mathbf{x})$ and

$$
\Pi(\overline{\mathbf{x}})=\Xi(\overline{\mathbf{x}}, \overline{\boldsymbol{\zeta}})=\Pi^{d}(\overline{\boldsymbol{\zeta}}) .
$$

Conversely, if $\overline{\mathbf{x}}$ is a solution to $(\mathcal{P})$, it must be in the form of (21) for critical solution $\overline{\boldsymbol{\varsigma}}$ of $\Pi^{d}(\boldsymbol{\varsigma})$.

This theorem has extensive applications in nonconvex analysis and global optimization [17]. In finite deformation theory, this complementary-dual principle solved a 50-years open problem [29]. Note that the feasible set $\mathcal{S}_{a}$ is not convex, in order to identity the extremality property of the critical solutions, we need to introduce the following subsets of $\mathcal{S}_{a}$ :

$$
\mathcal{S}_{a}^{+}=\left\{\boldsymbol{\varsigma} \in \mathcal{S}_{a} \mid G(\boldsymbol{\varsigma}) \succ 0\right\}, \mathcal{S}_{a}^{-}=\left\{\boldsymbol{\varsigma} \in \mathcal{S}_{a} \mid G(\boldsymbol{\varsigma}) \prec 0\right\} .
$$


Theorem 2 (Triality Theory). Suppose the $(\overline{\mathbf{x}}, \overline{\boldsymbol{\zeta}})$ is a critical point of $\Xi(\mathbf{x}, \boldsymbol{\varsigma})$. The critical solution $\overline{\mathbf{x}}$ is a unique global minimizer of $(\mathcal{P})$ if and only if $\overline{\boldsymbol{\varsigma}} \in \mathcal{S}_{a}^{+}$is a global maximizer of $\Pi^{d}(\boldsymbol{\varsigma})$ on $\mathcal{S}_{a}^{+}$, i.e.

$$
\Pi(\overline{\mathbf{x}})=\min _{\mathbf{x} \in \mathcal{X}_{a}^{\prime}} \Pi(\mathbf{x}) \Leftrightarrow \max _{\boldsymbol{\varsigma} \in \mathcal{S}_{a}^{+}} \Pi^{d}(\boldsymbol{\varsigma})=\Pi^{d}(\overline{\boldsymbol{\zeta}}) .
$$

If $\overline{\boldsymbol{\varsigma}} \in \mathcal{S}_{a}^{-}$, then $\overline{\boldsymbol{\varsigma}}$ is a local maximizer of $\Pi^{d}(\boldsymbol{\varsigma})$ on its neighborhood $\mathcal{S}_{o} \subset \mathcal{S}_{a}^{-}$ if and only if $\overline{\mathbf{x}}$ is a local maximizer of $(\mathcal{P})$ on its neighborhood $\mathcal{X}_{o} \subset \mathcal{X}_{a}$, i.e.

$$
\Pi(\overline{\mathbf{x}})=\max _{\mathbf{x} \in \mathcal{X}_{o}} \Pi(\mathbf{x}) \Leftrightarrow \max _{\boldsymbol{\varsigma} \in \mathcal{S}_{o}} \Pi^{d}(\boldsymbol{\varsigma})=\Pi^{d}(\overline{\boldsymbol{\varsigma}}) .
$$

If $\overline{\boldsymbol{\varsigma}} \in \mathcal{S}_{a}^{-}$and $\operatorname{dim} \mathcal{X}_{a}=\operatorname{dim} \mathcal{S}_{a}$, then $\overline{\boldsymbol{\varsigma}}$ is a local minimizer of $\Pi^{d}(\boldsymbol{\varsigma})$ on its neighborhood $\mathcal{S}_{o} \subset \mathcal{S}_{a}^{-}$if and only if $\overline{\mathbf{x}}$ is a local minimizer of $(\mathcal{P})$ on its neighborhood $\mathcal{X}_{o} \subset \mathcal{X}_{a}$, i.e.

$$
\Pi(\overline{\mathbf{x}})=\min _{\mathbf{x} \in \mathcal{X}_{o}} \Pi(\mathbf{x}) \Leftrightarrow \min _{\boldsymbol{\varsigma} \in \mathcal{S}_{o}} \Pi^{d}(\boldsymbol{\varsigma})=\Pi^{d}(\overline{\boldsymbol{\varsigma}}) .
$$

Remark 1. The saddle min-max duality theorem (23) was first proved by Gao and Strang in finite deformation theory [22], while the double-min and double-max duality statements were discovered in 1996.

The double-max duality statement (24) can be proved easily by the fact that

$$
\max _{\mathbf{x} \in \mathcal{X}_{o}} \max _{\boldsymbol{\varsigma} \in \mathcal{S}_{o}} \Xi(\mathbf{x}, \boldsymbol{\varsigma})=\max _{\boldsymbol{\varsigma} \in \mathcal{S}_{o}} \max _{\mathbf{x} \in \mathcal{X}_{o}} \Xi(\mathbf{x}, \boldsymbol{\varsigma}) \forall(\mathbf{x}, \boldsymbol{\varsigma}) \in \mathcal{X}_{o} \times \mathcal{S}_{o} \subset \mathcal{X}_{a} \times \mathcal{S}_{a}^{-} .
$$

The double-min duality statement (25) holds only under the condition $\operatorname{dim} \mathcal{X}_{a}=\operatorname{dim} \mathcal{S}_{a}$, which was an open problem discovered in 2003 [14, 15] and solved recently in [23]. If $\operatorname{dim} \mathcal{X}_{a} \neq \operatorname{dim} \mathcal{S}_{a}$, this double-min duality holds in a weak form (see [23]).

Based on the triality theory, the nonconvex minimization problem $(\mathcal{P})$ is equivalent (only if $\mathcal{S}_{a}^{+} \neq \emptyset$ ) to a concave maximization dual problem

$$
\left(\mathcal{P}^{d}\right): \max \left\{\Pi^{d}(\varsigma) \mid \varsigma \in \mathcal{S}_{a}^{+}\right\} .
$$

Although $\Pi^{d}(\boldsymbol{\varsigma})$ contains an inverse matrix of $\mathbf{G}(\boldsymbol{\varsigma})$, this canonical dual problem can be solved easily by some well-developed nonlinear optimization techniques (see [1], 40]) as long as $\mathcal{S}_{a}^{+}$contains at least one critical point of $\Pi^{d}(\boldsymbol{\sigma})$. Otherwise, a perturbation method will be discussed in the next section. 


\section{Example 1 (Fundamental idea of linear perturbation).}

To explain the theory, let us consider a very simple nonconvex optimization in $\mathbb{R}^{n}$ :

$$
\min \left\{\Pi(\mathbf{x})=\frac{1}{2} \alpha\left(\frac{1}{2}\|\mathbf{x}\|^{2}-\lambda\right)^{2}-\mathbf{x}^{T} \mathbf{f} \quad \forall \mathbf{x} \in \mathbb{R}^{n}\right\},
$$

where $\alpha, \lambda>0$ are given parameters. The criticality condition $\nabla P(\mathbf{x})=0$ leads to a nonlinear algebraic equation system in $\mathbb{R}^{n}$

$$
\alpha\left(\frac{1}{2}\|\mathbf{x}\|^{2}-\lambda\right) \mathbf{x}=\mathbf{f} .
$$

Clearly, to solve this nonlinear algebraic equation directly is difficult. Also traditional convex optimization theory can not be used to identify global minimizer. However, by the canonical dual transformation, this problem can be solved completely and easily. To do so, we let $\xi=\Lambda(\mathbf{x})=\frac{1}{2}\|\mathbf{x}\|^{2} \in \mathbb{R}$, which is an objective measure. Then, the nonconvex function $W(\mathbf{x})=$ $\frac{1}{2} \alpha\left(\frac{1}{2}\|\mathbf{x}\|^{2}-\lambda\right)^{2}$ can be written in canonical form $V(\xi)=\frac{1}{2} \alpha(\xi-\lambda)^{2}$. Its Legendre conjugate is given by $V^{*}(\varsigma)=\frac{1}{2} \alpha^{-1} \varsigma^{2}+\lambda \varsigma$, which is strictly convex. Thus, the total complementary function for this nonconvex optimization problem is

$$
\Xi(\mathbf{x}, \varsigma)=\frac{1}{2}\|\mathbf{x}\|^{2} \varsigma-\frac{1}{2} \alpha^{-1} \varsigma^{2}-\lambda \varsigma-\mathbf{x}^{T} \mathbf{f} .
$$

For a fixed $\varsigma \in \mathbb{R}$, the criticality condition $\nabla_{\mathbf{x}} \Xi(\mathbf{x})=0$ leads to

$$
\varsigma \mathbf{X}-\mathbf{f}=0 .
$$

For each $\varsigma \neq 0$, the equation (30) gives $\mathbf{x}=\mathbf{f} / \varsigma$ in vector form. Substituting this into the total complementary function $\Xi$, the canonical dual function can be easily obtained as

$$
\begin{aligned}
\Pi^{d}(\varsigma) & =\left\{\Xi(\mathbf{x}, \varsigma) \mid \nabla_{\mathbf{x}} \Xi(\mathbf{x}, \varsigma)=0\right\} \\
& =-\frac{\mathbf{f}^{T} \mathbf{f}}{2 \varsigma}-\frac{1}{2} \alpha^{-1} \varsigma^{2}-\lambda \varsigma, \quad \forall \varsigma \neq 0 .
\end{aligned}
$$

The critical point of this canonical function is obtained by solving the following dual algebraic equation

$$
\left(\alpha^{-1} \varsigma+\lambda\right) \varsigma^{2}=\frac{1}{2} \mathbf{f}^{T} \mathbf{f}
$$


For any given parameters $\alpha, \lambda$ and the vector $\mathbf{f} \in \mathbb{R}^{n}$, this cubic algebraic equation has at most three real roots satisfying $\varsigma_{1} \geq 0 \geq \varsigma_{2} \geq \varsigma_{3}$, and each of these roots leads to a critical point of the nonconvex function $P(\mathbf{x})$, i.e., $\mathbf{x}_{i}=\mathbf{f} / \varsigma_{i}, i=1,2,3$. By the fact that $\varsigma_{1} \in \mathcal{S}_{a}^{+}=\{\varsigma \in \mathbb{R} \mid \varsigma>0\}, \varsigma_{2,3} \in$ $\mathcal{S}_{a}^{-}=\{\varsigma \in \mathbb{R} \mid \varsigma<0\}$, then Theorem 2 tells us that $\mathbf{x}_{1}$ is a global minimizer of $\Pi(\mathbf{x}), \mathbf{x}_{3}$ is a local maximizer of $\Pi(\mathbf{x})$, while $\mathbf{x}_{2}$ is a local minimizer if $n=1$ (see Fig. 1). If we choose $n=1, \quad \alpha=1, \lambda=2$, and $f=\frac{1}{2}$, the primal function and canonical dual function are shown in Fig. 1 (a), where, $x_{1}=2.11491$ is global minimizer of $\Pi(\mathbf{x}), \varsigma_{1}=0.236417$ is global maximizer of $\Pi^{d}(\boldsymbol{\varsigma})$, and $\Pi\left(x_{1}\right)=-1.02951=\Pi^{d}\left(\varsigma_{1}\right)$ (see the two black dots). Also it is easy to verify that $x_{2}$ is a local minimizer, while $x_{3}$ is a local maximizer.

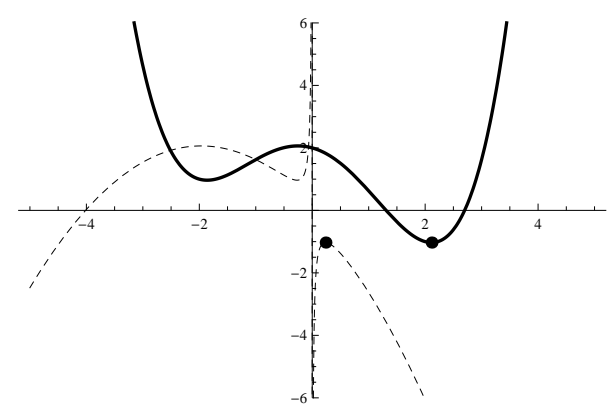

(a) $f=0.5$

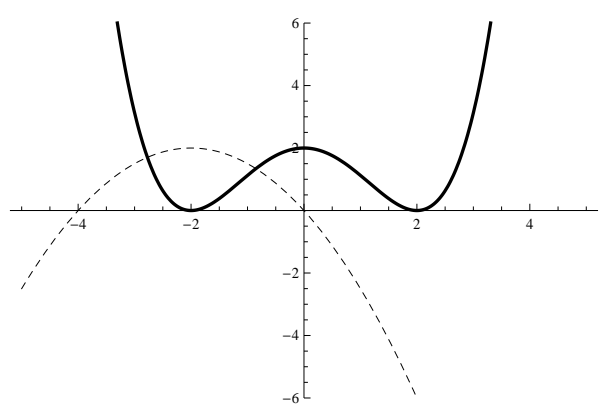

(b) $f=0$

Figure 1: Graphs of the primal function $\Pi(\mathbf{x})$ (solid) and its canonical dual $\Pi^{d}(\varsigma)$ (dashed).

If we let $\mathbf{f}=0$, the graph of $\Pi(\mathbf{x})$ is symmetric (i.e. the so-called doublewell potential or the Mexican hat for $n=2$ [15]) with infinite number of global minimizers satisfying $\|\mathbf{x}\|^{2}=2 \lambda$. In this case, the canonical dual $\Pi^{d}(\varsigma)=-\frac{1}{2} \alpha^{-1} \varsigma^{2}-\lambda \varsigma$ is strictly concave with only one critical point (local maximizer) $\varsigma_{3}=-\alpha \lambda \in \mathcal{S}_{a}^{-}$(for $\alpha, \lambda>0$ ). The corresponding solution $\mathbf{x}_{3}=\mathbf{f} / \varsigma_{3}=0$ is a local maximizer. By the canonical dual equation (32) we have $\varsigma_{1}=\varsigma_{2}=0$ located on the boundary of $\mathcal{S}_{a}^{+}$, which corresponding to the two global minimizers $x_{1,2}= \pm \sqrt{2 \lambda}$ for $n=1$, see Fig. 1 (b). This is exactly the example of one sensor $\mathbf{x}=(x, 0) \in \mathbb{R}^{2}$ and two anchors $\mathbf{a}_{1,2}=(0, \pm a)$ with $e_{1}=e_{2}=b$. Due to symmetry $(f=0)$, the problem $(\mathcal{P})$ has two possible solutions $\mathbf{x}_{1,2}=\left(x_{1,2}, 0\right)$ with $\lambda=\frac{1}{2}\left(b^{2}-a^{2}\right)$. 
This simple example shows a fundament issue in global optimization, i.e., the optimal solutions of a nonconvex problem depends sensitively on the linear term (input) f. Geometrically speaking, the objective function $W(\mathbf{B x})$ in $\Pi(\mathbf{x})$ possesses certain symmetry. If there is no linear term (subjective function) in $\Pi(\mathbf{x})$, the nonconvex problem usually has more than one global minimizer due to the symmetry. Traditional direct approaches and the popular SDP method are usually failed to deal with this situation. By the canonical duality theory, we understand that in this case the canonical dual function has no critical point in $\mathcal{S}_{a}^{+}$. Therefore, by adding a linear perturbation $\mathbf{f}$ to destroy this symmetry, the canonical duality theory can be used to solve the nonconvex problems to obtain one of global optimal solutions. This idea was originally from Gao's work (1996) on post-buckling analysis of large deformed beam [11], where the triality theorem was first proposed [12]. The potential energy of this beam model is a double-well function, similar to this sensor example, without lateral force or imperfection, the beam could have two buckling states (corresponding to two minimizers) and one un-buckled state (local maximizer). Later on (2008) in the Gao and Ogden work on analytical solutions in phase transformation [18], they further discovered that the nonconvex system has no phase transition unless the force distribution $f(x)$ vanished at certain points. They also discovered that if force field $f(x)$ changes dramatically, all the Newton type direct approaches failed even to find any local minimizer. The linear perturbation method has been used successfully for solving global optimization problems [6, 37, 39, 43].

\section{Application to Sensor Network Localization Problem}

Now let us tern our attention for solving the general sensor network optimization problem $\left(\mathcal{P}_{1}\right)$. For convenience, we transfer variables from matrix 
to vectors, and let

$$
\begin{aligned}
\mathbf{y}= & {\left[x_{1}^{1} \cdots x_{1}^{d} \cdots x_{n}^{1} \cdots x_{n}^{d}\right]^{T} \in \mathbb{R}^{n d}: \text { Locations of sensors (variables), } } \\
\mathbf{W}= & {\left[w_{11} \cdots w_{1 n} \cdots w_{n 1} \cdots w_{n n}\right]^{T} \in \mathbb{R}^{n n}: } \\
& \text { Weights for the optimization problem }\left(\mathcal{P}_{1}\right), \\
\mathbf{Q}= & {\left[q_{11} \cdots q_{1 m} \cdots q_{n 1} \cdots q_{n m}\right]^{T} \in \mathbb{R}^{n m}: } \\
& \text { Weights for the optimization problem }\left(\mathcal{P}_{1}\right), \\
\mathbf{a}= & {\left[\sum_{\alpha=1}^{d}\left(a_{1}^{\alpha}\right)^{2}, \cdots, \sum_{\alpha=1}^{d}\left(a_{m}^{\alpha}\right)^{2}\right]^{T}: } \\
& \text { Sums of squares of anchors, } \\
\mathbf{d}= & {\left[d_{11}^{2} \cdots d_{1 n}^{2} \cdots d_{n 1}^{2} \cdots d_{n n}^{2}\right]^{T} \in \mathbb{R}^{n n}: \text { Squares of distances between sensors, } } \\
\mathbf{e}= & {\left[e_{11}^{2} \cdots e_{1 m}^{2} \cdots e_{n 1}^{2} \cdots e_{n m}^{2}\right]^{T} \in \mathbb{R}^{n m}: }
\end{aligned}
$$

Squares of distances between sensors and anchors.

Then, Problem $\left(\mathcal{P}_{1}\right)$ can be written in a vector form given below.

$$
\begin{aligned}
(\mathcal{P}): \min & \left\{\Pi(\mathbf{y})=\sum_{(i, j) \in A_{d}} \frac{1}{2} w_{i j}\left(\mathbf{y}^{T} D_{i j} \mathbf{y}-d_{i j}^{2}\right)^{2}\right. \\
& \left.+\sum_{(i, k) \in A_{e}} \frac{1}{2} q_{i k}\left(\mathbf{y}^{T} E_{i k} \mathbf{y}-2 A_{i k}^{T} \mathbf{y}+\sum_{\alpha=1}^{d}\left(a_{i k}^{\alpha}\right)^{2}-e_{i k}^{2}\right)^{2}\right\},
\end{aligned}
$$

where $A_{i k}$ and $a_{i k}^{\alpha}$ are components of the anchors $\mathbf{a}_{k}$ obtained from the expansion of $\left\|\mathbf{x}_{i}-\mathbf{a}_{k}\right\|^{2}$ in equation (3), $E_{i k} \in \mathbb{R}^{n d \times n d}$ is a diagonal matrix defined by

$$
E_{i k}=\left[\begin{array}{ccc}
0 & 0 & 0 \\
0 & I_{i k} & 0 \\
0 & 0 & 0
\end{array}\right],
$$

with $I_{i k} \in \mathbb{R}^{d \times d}$ being the identity matrix corresponding to sensor $i$ and anchor $k$, so that the $(1,1)$ entry of $I_{i k}$ coincides with the $(i, k)$ entry of $E_{i k}$. Similarly, $D_{i j}$ is an $n d \times n d$ matrix defined by

$$
D_{i j}=\left[\begin{array}{ccccc}
0 & 0 & 0 & 0 & 0 \\
0 & I_{i i} & 0 & -I_{i j} & 0 \\
0 & 0 & 0 & 0 & 0 \\
0 & -I_{j i} & 0 & I_{j j} & 0 \\
0 & 0 & 0 & 0 & 0
\end{array}\right]
$$


with $I_{i i}, I_{j j}, I_{i j}, I_{j i} \in \mathbb{R}^{d \times d}$ being the identity matrices, so that the $(1,1)$ entry of $I_{i i}$ coincides with the $(i, i)$ entry of the matrix $D_{i j}$. For $I_{j j}, I_{i j}, I_{j i}$, they are defined similarly. Let

$$
\begin{aligned}
\xi_{i j} & =\Lambda_{i j}(\mathbf{y})=\mathbf{y}^{T} D_{i j} \mathbf{y} \\
\epsilon_{i k} & =\Lambda_{i k}(\mathbf{y})=\mathbf{y}^{T} E_{i k} \mathbf{y}-2 A_{i k}^{T} \mathbf{y},
\end{aligned}
$$

where $\Lambda_{i j}$ and $\Lambda_{i k}$ are, respectively, geometrical operators from $\mathbb{R}^{\text {nd }}$ into

$$
\mathcal{E}_{d}=\left\{\boldsymbol{\xi} \in \mathbb{R}^{n n} \mid \xi_{i j} \geq 0, \xi_{i j}=0 \text { if } i=j\right\}
$$

and

$$
\mathcal{E}_{e}=\left\{\boldsymbol{\epsilon} \in \mathbb{R}^{m n} \mid \epsilon_{i k} \geq 0\right\} .
$$

By introducing quadratic functions $V_{\xi}: \mathcal{E}_{d} \rightarrow \mathbb{R}$ and $V_{\epsilon}: \mathcal{E}_{e} \rightarrow \mathbb{R}$ such that

$$
V_{\xi}\left(\xi_{i j}\right)=\frac{1}{2} \sum_{(i, j) \in \mathcal{A}_{d}} w_{i j}\left(\xi_{i j}-d_{i j}^{2}\right)^{2}
$$

and

$$
V_{\epsilon}\left(\epsilon_{i k}\right)=\frac{1}{2} \sum_{(i, k) \in \mathcal{A}_{e}} q_{i k}\left(\epsilon_{i k}+\sum_{\alpha=1}^{d}\left(a_{i k}^{\alpha}\right)^{2}-e_{i k}^{2}\right)^{2}
$$

Problem $(\mathcal{P})$ can then be reformulated in the canonical form given below:

$$
(\mathcal{P}): \min \left\{\Pi(\mathbf{y})=V_{\xi}\left(\Lambda_{i j}(\mathbf{y})\right)+V_{\epsilon}\left(\Lambda_{i k}(\mathbf{y})\right) \mid \mathbf{y} \in \mathbb{R}^{n d}\right\} .
$$

Note that the function $V_{\xi}\left(\xi_{i j}\right)$ and $V_{\epsilon}\left(\epsilon_{i k}\right)$ are both convex. Then the following duality relations are invertible

$$
\varsigma_{i j}=\nabla V_{\xi}\left(\xi_{i j}\right)=w_{i j}\left(\xi_{i j}-d_{i j}^{2}\right), \quad(i, j) \in \mathcal{A}_{d},
$$

and

$$
\sigma_{i k}=\nabla V_{\epsilon}\left(\epsilon_{i k}\right)=q_{i k}\left(\epsilon_{i k}+\sum_{\alpha=1}^{d}\left(a_{i k}^{\alpha}\right)^{2}-e_{i k}^{2}\right), \quad(i, k) \in \mathcal{A}_{e},
$$

where $\varsigma_{i j}$ and $\sigma_{i k}$ are dual variables. Let $\mathcal{S}_{d}$ be the range of the duality mapping $\varsigma_{i j}=\nabla V_{\xi}\left(\xi_{i j}\right)$, and let $\mathcal{S}_{e}$ be the range of the duality mapping 
$\sigma_{i k}=\nabla V_{\epsilon}\left(\epsilon_{i k}\right)$. Then, for any given $\boldsymbol{\varsigma} \in \mathcal{S}_{d}$ and $\boldsymbol{\sigma} \in \mathcal{S}_{e}$, the Legendre conjugate $V_{\xi}^{*}$ and $V_{\epsilon}^{*}$ can be uniquely defined by

$$
\begin{aligned}
V_{\xi}^{*}\left(\varsigma_{i j}\right) & =\operatorname{sta}\left\{\sum_{(i, j) \in \mathcal{A}_{d}} \xi_{i j} \varsigma_{i j}-V_{\xi}\left(\xi_{i j}\right) \mid \xi_{i j} \in \mathcal{V}_{d}\right\} \\
& =\sum_{(i, j) \in \mathcal{A}_{d}}\left(\frac{1}{2} w_{i j}^{-1} \varsigma_{i j}^{2}+d_{i j}^{2} \varsigma_{i j}\right)
\end{aligned}
$$

and

$$
\begin{aligned}
V_{\epsilon}^{*}\left(\sigma_{i k}\right) & =\operatorname{sta}\left\{\sum_{(i, k) \in \mathcal{A}_{e}} \epsilon_{i k} \sigma_{i k}-V_{\epsilon}\left(\epsilon_{i k}\right) \mid \epsilon_{i k} \in \mathcal{V}_{e}\right\} \\
& =\sum_{(i, k) \in \mathcal{A}_{e}}\left[\frac{1}{2} q_{i k}^{-1} \sigma_{i k}^{2}+\left(e_{i k}^{2}-\sum_{\alpha=1}^{d}\left(a_{i k}^{\alpha}\right)^{2}\right) \sigma_{i k}\right]
\end{aligned}
$$

Clearly, $(\boldsymbol{\xi}, \boldsymbol{\varsigma})$ and $(\boldsymbol{\epsilon}, \boldsymbol{\sigma})$ form a canonical duality pair (see [13]). The following canonical duality relations hold on both $\mathcal{E}_{d} \times \mathcal{S}_{d}$ and $\mathcal{E}_{e} \times \mathcal{S}_{e}$

$$
\begin{aligned}
\boldsymbol{\varsigma} & =\nabla V_{\xi}(\boldsymbol{\xi}) \Leftrightarrow \boldsymbol{\xi}=\nabla V_{\xi}^{*}(\boldsymbol{\varsigma}) \Leftrightarrow\langle\boldsymbol{\xi} ; \boldsymbol{\varsigma}\rangle=V_{\xi}(\boldsymbol{\xi})+V_{\xi}^{*}(\boldsymbol{\varsigma}), \\
\boldsymbol{\sigma} & =\nabla V_{\epsilon}(\boldsymbol{\epsilon}) \Leftrightarrow \boldsymbol{\epsilon}=\nabla V_{\epsilon}^{*}(\boldsymbol{\sigma}) \Leftrightarrow\langle\boldsymbol{\epsilon} ; \boldsymbol{\sigma}\rangle=V_{\epsilon}(\boldsymbol{\epsilon})+V_{\epsilon}^{*}(\boldsymbol{\sigma}),
\end{aligned}
$$

respectively. So the generalized complementary function $([13])$ is given by

$$
\begin{aligned}
\Xi(\mathbf{y}, \boldsymbol{\varsigma}, \boldsymbol{\sigma})= & \sum_{(i, j) \in A_{d}} \Lambda_{i j}(\mathbf{y}) \varsigma_{i j}-V_{\xi}^{*}(\boldsymbol{\varsigma})+\sum_{(i, k) \in A_{e}} \Lambda_{i k}(\mathbf{y}) \sigma_{i k}-V_{\epsilon}^{*}(\boldsymbol{\sigma}) \\
= & \frac{1}{2} \mathbf{y}^{T} \mathbf{G}(\boldsymbol{\varsigma}, \boldsymbol{\sigma}) \mathbf{y}-\mathbf{F}^{T}(\boldsymbol{\sigma}) \mathbf{y}-\frac{1}{2}\left(\mathbf{W}^{-1}\right)^{T}(\boldsymbol{\varsigma} \circ \boldsymbol{\varsigma}) \\
& -\frac{1}{2}\left(\mathbf{Q}^{-1}\right)^{T}(\boldsymbol{\sigma} \circ \boldsymbol{\sigma})-\mathbf{d}^{T} \boldsymbol{\varsigma}+\mathbf{a}^{T} \boldsymbol{\sigma}-\mathbf{e}^{T} \boldsymbol{\sigma}
\end{aligned}
$$

where $\mathbf{s} \circ \mathbf{t}:=\left[s_{1} t_{1}, s_{2} t_{2}, \cdots, s_{n} t_{n}\right]^{T}$ denotes the Hadamard product of any two vectors $\mathbf{s}, \mathbf{t} \in \mathbb{R}^{n}$,

$$
\mathbf{F}(\boldsymbol{\sigma})=\left[\sum_{k=1}^{m} 2 a_{k}^{1} \sigma_{1 k} \cdots \sum_{k=1}^{m} 2 a_{k}^{d} \sigma_{1 k} \cdots \sum_{k=1}^{m} 2 a_{k}^{1} \sigma_{n k} \cdots \sum_{k=1}^{m} 2 a_{k}^{d} \sigma_{n k}\right]^{T}
$$




$$
\mathbf{G}(\boldsymbol{\varsigma}, \boldsymbol{\sigma})=2\left(\operatorname{Diag}\left(F_{1}(\boldsymbol{\varsigma})\right)+\operatorname{Diag}\left(F_{2}(\boldsymbol{\sigma})\right)+G_{3}(\boldsymbol{\varsigma})\right),
$$

with

$$
\begin{gathered}
F_{1}(\boldsymbol{\varsigma})=\left[\begin{array}{c}
\sum_{i=1}^{n} \varsigma_{1 i} \\
\vdots \\
\sum_{i=1}^{n} \varsigma_{1 i} \\
\vdots \\
\sum_{i=1}^{n} \varsigma_{n i} \\
\vdots \\
\sum_{i=1}^{n} \varsigma_{n i}
\end{array}\right], F_{2}(\boldsymbol{\sigma})=\left[\begin{array}{c}
\sum_{k=1}^{m} \sigma_{1 k} \\
\vdots \\
\sum_{k=1}^{m} \sigma_{1 k} \\
\vdots \\
\sum_{k=1}^{m} \sigma_{n k} \\
\vdots \\
\sum_{k=1}^{m} \sigma_{n k}
\end{array}\right], \\
G_{3}(\boldsymbol{\varsigma})=\left[\begin{array}{ccc}
-\varsigma_{11} I_{11} & \cdots & -\varsigma_{1 n} I_{1 n} \\
\vdots & \vdots & \vdots \\
-\varsigma_{n 1} I_{n 1} & \cdots & -\varsigma_{n n} I_{n n}
\end{array}\right] .
\end{gathered}
$$

where the notation Diag $\left(F_{1}\right)$ represents a diagonal matrix with $F_{1 i}, i=$ $1, \cdots, n$ being its diagonal elements. For a fixed $\varsigma \in \mathcal{S}_{d}$ and $\boldsymbol{\sigma} \in \mathcal{S}_{e}$, the criticality condition $\nabla_{\mathbf{y}} \Xi(\mathbf{y}, \boldsymbol{\varsigma}, \boldsymbol{\sigma})=0$ leads to the following canonical equilibrium equation:

$$
\mathbf{G}(\boldsymbol{\varsigma}, \boldsymbol{\sigma}) \mathbf{y}-\mathbf{F}(\boldsymbol{\sigma})=0 .
$$

Substitute the solution of this equation into (41), the canonical dual function can be formulated as:

$$
\begin{aligned}
\Pi^{d}(\boldsymbol{\varsigma}, \boldsymbol{\sigma})= & -\frac{1}{2} \mathbf{F}(\boldsymbol{\sigma})^{T} \mathbf{G}^{-1}(\boldsymbol{\varsigma}, \boldsymbol{\sigma}) \mathbf{F}(\boldsymbol{\sigma})-\frac{1}{2}\left(\mathbf{W}^{-1}\right)^{T}(\boldsymbol{\varsigma} \circ \boldsymbol{\varsigma}) \\
& -\frac{1}{2}\left(\mathbf{Q}^{-1}\right)^{T}(\boldsymbol{\sigma} \circ \boldsymbol{\sigma})-\mathbf{d}^{T} \boldsymbol{\varsigma}+\mathbf{a}^{T} \boldsymbol{\sigma}-\mathbf{e}^{T} \boldsymbol{\sigma}
\end{aligned}
$$

where

$$
\mathbf{F}(\boldsymbol{\sigma})=\left[\sum_{k=1}^{m} 2 a_{k}^{1} \sigma_{1 k} \cdots \sum_{k=1}^{m} 2 a_{k}^{d} \sigma_{1 k} \cdots \sum_{k=1}^{m} 2 a_{k}^{1} \sigma_{n k} \cdots \sum_{k=1}^{m} 2 a_{k}^{d} \sigma_{n k}\right]^{T}
$$

and

$$
\begin{aligned}
\mathbf{W}^{-1} & =\left[\frac{1}{w_{11}} \cdots \frac{1}{w_{1 n}} \cdots \frac{1}{w_{n 1}} \cdots \frac{1}{w_{n n}}\right]^{T} \\
\mathbf{Q}^{-1} & =\left[\frac{1}{q_{11}} \cdots \frac{1}{q_{1 m}} \cdots \frac{1}{q_{n 1}} \cdots \frac{1}{q_{n m}}\right]^{T} .
\end{aligned}
$$


Therefore, the canonical dual problem can be written in the form given below.

$$
\left(\mathcal{P}^{d}\right): \operatorname{sta}\left\{\Pi^{d}(\boldsymbol{\varsigma}, \boldsymbol{\sigma}) \mid \boldsymbol{\varsigma} \in \mathcal{S}_{d}, \boldsymbol{\sigma} \in \mathcal{S}_{e}\right\} .
$$

By Theorem 1, we have following results:

Theorem 3. Problem $\left(\mathcal{P}^{d}\right)$ is a canonical dual of the primal problem $(\mathcal{P})$ in the sense that if $(\overline{\boldsymbol{\kappa}}, \overline{\boldsymbol{\sigma}})$ is a critical point of $\left(\mathcal{P}^{d}\right)$, then

$$
\overline{\mathbf{y}}=\mathbf{G}^{-1}(\overline{\boldsymbol{\zeta}}, \overline{\boldsymbol{\sigma}}) \mathbf{F}(\overline{\boldsymbol{\sigma}})
$$

is a critical point of $(\mathcal{P})$ and

$$
\Pi(\overline{\mathbf{y}})=\Pi^{d}(\overline{\boldsymbol{\zeta}}, \overline{\boldsymbol{\sigma}})
$$

Theorem 3 shows that the nonconvex primal problem $(\mathcal{P})$ is equivalent to its canonical dual problem $\left(\mathcal{P}^{d}\right)$ with zero duality gap, and the solution of $(\mathcal{P})$ can be analytically expressed by (46) in terms of the canonical dual variables. The global minimizer can be identified by the saddle min-max duality theorem (23). In this case, the feasible space $\mathcal{S}_{a}^{+}$should be

$$
\mathcal{S}_{a}^{+}=\left\{(\boldsymbol{\varsigma}, \boldsymbol{\sigma}) \in \mathcal{S}_{d} \times \mathcal{S}_{e} \mid \mathbf{G}(\boldsymbol{\varsigma}, \boldsymbol{\sigma}) \succ 0\right\} .
$$

Based on the triality theory, the nonconvex primal problem $(\mathcal{P})$ is equivalent to the following canonical dual problem:

$$
\left(\mathcal{P}_{\max }^{d}\right): \max \left\{\Pi^{d}(\boldsymbol{\varsigma}, \boldsymbol{\sigma}) \mid(\boldsymbol{\varsigma}, \boldsymbol{\sigma}) \in \mathcal{S}_{a}^{+}\right\} .
$$

Theorem 4. If $(\overline{\boldsymbol{\varsigma}}, \overline{\boldsymbol{\sigma}}) \in \mathcal{S}_{a}^{+}$is a critical point of the canonical dual function $\Pi^{d}(\overline{\boldsymbol{\kappa}}, \overline{\boldsymbol{\sigma}})$, then it is a unique solution of $\left(\mathcal{P}_{\max }^{d}\right)$, the vector $\overline{\mathbf{y}}=\mathbf{G}^{-1}(\overline{\boldsymbol{\kappa}}, \overline{\boldsymbol{\sigma}}) F(\overline{\boldsymbol{\sigma}})$ is a unique global optimal solution to the nonconvex sensor network optimization problem $(\mathcal{P})$, and

$$
\Pi(\overline{\mathbf{y}})=\min _{\mathbf{y} \in \mathbb{R}^{n d}} \Pi(\mathbf{y})=\max _{(\boldsymbol{\varsigma}, \boldsymbol{\sigma}) \in \mathcal{S}_{a}^{+}} \Pi^{d}(\boldsymbol{\varsigma}, \boldsymbol{\sigma})=\Pi^{d}(\overline{\boldsymbol{\zeta}}, \overline{\boldsymbol{\sigma}}) .
$$

Proof. First, we need to prove the convexity of $\mathcal{S}_{a}^{+}$. We let $\boldsymbol{\xi}^{*}=(\boldsymbol{\varsigma}, \boldsymbol{\sigma})$. For any given $\boldsymbol{\xi}_{1}^{*}, \boldsymbol{\xi}_{2}^{*} \in \mathcal{S}_{a}^{+}$, we should have

$$
\theta \mathbf{G}\left(\boldsymbol{\xi}_{1}^{*}\right) \succ 0, \quad(1-\theta) \mathbf{G}\left(\boldsymbol{\xi}_{2}^{*}\right) \succ 0 \quad \forall \theta \in[0,1] .
$$


Therefore,

$$
\theta \mathbf{G}\left(\boldsymbol{\xi}_{1}^{*}\right)+(1-\theta) \mathbf{G}\left(\boldsymbol{\xi}_{2}^{*}\right)=\mathbf{G}\left(\theta \boldsymbol{\xi}_{1}^{*}+(1-\theta) \boldsymbol{\xi}_{2}^{*}\right) \succ 0 \quad \forall \theta \in[0,1] .
$$

This shows that $\mathcal{S}_{a}^{+}$is convex.

By the fact that the total complementary function $\Xi\left(\mathbf{y}, \boldsymbol{\xi}^{*}\right)$ is a saddle function on $\mathbb{R}^{n d} \times \mathcal{S}_{a}^{+}$, the classical saddle min-max duality theory (cf. [9], page 57, or [13], page 39) leads to

$$
\min \Pi(\mathbf{y})=\min _{\mathbf{y} \in \mathbb{R}^{n d}} \max _{\boldsymbol{\xi}^{*} \in \mathcal{S}_{a}^{+}} \Xi\left(\mathbf{y}, \boldsymbol{\xi}^{*}\right)=\max _{\boldsymbol{\xi}^{*} \in \mathcal{S}_{a}^{+}} \min _{\mathbf{y} \in \mathbb{R}^{n d}} \Xi\left(\mathbf{y}, \boldsymbol{\xi}^{*}\right)=\max _{\boldsymbol{\xi}^{*} \in \mathcal{S}_{a}^{+}} \Pi^{d}\left(\boldsymbol{\xi}^{*}\right) .
$$

Therefore, by the complementary-dual principle, the critical point $\overline{\mathbf{y}} \in \mathbb{R}^{\text {nd }}$ of $\Xi$ is a global min of $\Pi(\mathbf{y})$ if and only if the associated critical point $\overline{\boldsymbol{\xi}}^{*}$ is a global max of $\Pi^{d}\left(\boldsymbol{\xi}^{*}\right)$ on $\mathcal{S}_{a}^{+}$. Since $\Xi\left(\mathbf{y}, \boldsymbol{\xi}^{*}\right)$ is strictly convex in $\mathbf{y}$ and concave in $\boldsymbol{\xi}^{*}$ on $\mathbb{R}^{n d} \times \mathcal{S}_{a}^{+}$, its saddle point is unique.

Remark 2 (NP-Hard Problems and Perturbations). It is known that the sensor localization problem is NP-hard only in the worst case (see [2]). However, what is the worst case is not clear to general problems. By the canonical duality theory we know that for a large class of nonconvex and integer optimization problems, as long as their canonical dual problems or perturbed forms have critical points in the dual feasible domain $S_{a}^{+}$, these challenging problems can be solved easily by convex optimization techniques (see [17]). Otherwise, these problems could be NP-hard, which is a conjecture proposed by Gao in 2007 [16].

By the fact that $\Pi^{d}(\boldsymbol{\varsigma})$ is concave on the open set $\mathcal{S}_{a}^{+}$, the canonical dual problem $\left(\mathcal{P}^{d}\right)$ may have no critical point in $\mathcal{S}_{a}^{+}$. It is our understanding that a NP-hard optimization problem usually possesses certain symmetry in its modeling if the primal problem has more than one global minimal solution. The main idea of the linear perturbation is to destroy this symmetry such that the associated perturbed problem has a unique solution. This method is wellknown in engineering mechanics and was introduced to global optimization by the authors in 2008 for solving quadratic equations via least squares method [37]. Recently, this method has been used successfully to solve a NP-complete max-cut problem [42] as well as some challenging problems in global optimization (see [6, 31]). For complex systems, how to correctly chose the linear perturbation vector $\boldsymbol{\delta}$ is still an open problem. Therefore, some high-order 
perturbation methods proposed in 2010 for solving certain NP-hard nonconvex/integer optimization problems [19]. Particularly, a quadratic perturbation method can be suggested as the following:

$$
\min _{\mathbf{y}} \max _{(\boldsymbol{\varsigma}, \boldsymbol{\sigma}) \in \mathcal{S}_{\mu_{k}}^{+}}\left\{\Xi_{k}(\mathbf{y}, \boldsymbol{\varsigma}, \boldsymbol{\sigma})=\Xi(\mathbf{y}, \boldsymbol{\varsigma}, \boldsymbol{\sigma})+\frac{1}{2} \rho_{k}\left\|\mathbf{y}-\mathbf{y}_{k}\right\|^{2}-\langle\mathbf{y}, \boldsymbol{\delta}\rangle\right\}
$$

where $\rho_{k}>0$ is a given parameter, $\mathbf{y}_{k} \in \mathbb{R}^{n d}$ is a given vector (previous solution in iteration process), $\boldsymbol{\delta} \in \mathbb{R}^{\text {nd }}$ is a given linear perturbation vector, $\mathcal{S}_{\mu_{k}}^{+}$is a relaxed canonical dual feasible space defined by

$$
\mathcal{S}_{\mu_{k}}^{+}=\left\{(\boldsymbol{\varsigma}, \boldsymbol{\sigma}) \in \mathcal{S}_{d} \times \mathcal{S}_{e} \mid \mathbf{G}(\boldsymbol{\varsigma}, \boldsymbol{\sigma})+\mu_{k} \mathbf{I} \succeq 0\right\}
$$

where $0<\mu_{k}<\rho_{k}$ is given relaxation parameter.

By the fact that the perturbed total complementary function $\Xi_{k}(\mathbf{y}, \boldsymbol{\varsigma}, \boldsymbol{\sigma})$ is strictly convex in $\mathbf{y} \in \mathbb{R}^{\text {nd }}$ and strictly concave in $(\boldsymbol{\varsigma}, \boldsymbol{\sigma})$ on the closed convex set $\mathcal{S}_{\mu_{k}}^{+}$, an effective canonical primal-dual algorithm can be developed for solving the saddle min-max problem (51).

\section{Numerical Simulations}

In this section, we will first look at a simple case of a network with four anchors and two sensors. The locations of the anchors are known while the locations of the sensors are to be determined. The linear perturbation method will be used to show how the symmetry can be destroyed such that this small scale network localization problem can be solved nicely. We then move on to formulate more general sensor networks by randomly generated test problems. The so-called root mean square distance (RMSD) will be used to measure the accuracy of the estimated positions.

\subsection{A four-anchor sensor network localization problem}

Consider the sensor network problem with two sensors and four anchors, as shown in Fig 2. Let $\mathbf{x}_{1}=\left[x_{1}^{1}, x_{1}^{2}\right]^{T}, \mathbf{x}_{2}=\left[x_{2}^{1}, x_{2}^{2}\right]^{T} \in \mathbb{R}^{2}$ denote the locations of the unknown sensors and let $\mathbf{a}_{i}, i=3,4,5,6$, denote the locations of the four known anchors. The sensor network location problem is to solve the following system of nonlinear equations:

$$
\begin{aligned}
& \left\|\mathbf{x}_{1}-\mathbf{x}_{2}\right\|=d_{12},\left\|\mathbf{x}_{1}-\mathbf{a}_{3}\right\|=e_{13},\left\|\mathbf{x}_{1}-\mathbf{a}_{4}\right\|=e_{14}, \\
& \left\|\mathbf{x}_{2}-\mathbf{a}_{5}\right\|=e_{25},\left\|\mathbf{x}_{2}-\mathbf{a}_{6}\right\|=e_{26},
\end{aligned}
$$




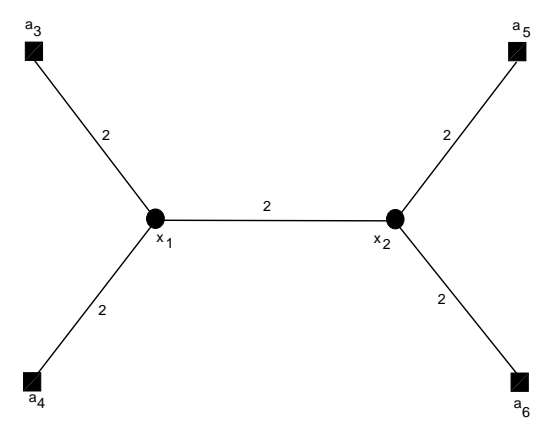

Figure 2: Sensor network with two sensors and four anchors.

where

$$
\begin{aligned}
& d_{12}=e_{13}=e_{14}=e_{25}=e_{26}=2, \\
& \mathbf{a}_{3}=[-2, \sqrt{3}=1.7321]^{T}, \quad \mathbf{a}_{4}=[-2,-\sqrt{3}=-1.7321]^{T}, \\
& \mathbf{a}_{5}=[2, \sqrt{3}=1.7321]^{T}, \quad \mathbf{a}_{6}=[2,-\sqrt{3}=-1.7321]^{T} .
\end{aligned}
$$

Let $\mathbf{y}=\left[x_{1}^{1} x_{1}^{2} x_{2}^{1} x_{2}^{2}\right]^{T} \in \mathbb{R}^{4}$.

Then, the fourth-order polynomial $\Pi(\mathbf{y})$ is given by

$$
\begin{aligned}
\Pi(\mathbf{y}) & =\frac{1}{2}\left(\left(x_{1}^{1}-x_{2}^{1}\right)^{2}+\left(x_{1}^{2}-x_{2}^{2}\right)^{2}-2^{2}\right)^{2}+\frac{1}{2}\left(\left(x_{1}^{1}+2\right)^{2}+\left(x_{1}^{2}-\sqrt{3}\right)^{2}-2^{2}\right)^{2} \\
& +\frac{1}{2}\left(\left(x_{1}^{1}+2\right)^{2}+\left(x_{1}^{2}+\sqrt{3}\right)^{2}-2^{2}\right)^{2}+\frac{1}{2}\left(\left(x_{2}^{1}-2\right)^{2}+\left(x_{2}^{2}-\sqrt{3}\right)^{2}-2^{2}\right)^{2} \\
& +\frac{1}{2}\left(\left(x_{2}^{1}-2\right)^{2}+\left(x_{2}^{2}+\sqrt{3}\right)^{2}-2^{2}\right)^{2} .
\end{aligned}
$$

To solve this symmetrical sensor network problem $\left(\mathcal{P}_{0}\right)$, we use a linear perturbation method (see [37])

$$
\left(\mathcal{P}_{\delta}\right): \min \left\{\Pi_{\delta}(\mathbf{y})=\Pi(\mathbf{y})-\boldsymbol{\delta}^{T} \mathbf{y} \mid \mathbf{y} \in \mathbb{R}^{4}\right\},
$$

where $\boldsymbol{\delta}=\left[\begin{array}{llll}\delta_{1}^{1} & \delta_{1}^{2} & \delta_{2}^{1} & \delta_{2}^{2}\end{array}\right]^{T} \geq 0$ is a given perturbation vector. On the canonical dual feasible space $\mathcal{S}_{a}$ defined by

$$
\mathcal{S}_{a}=\left\{\left(\varsigma_{12}, \sigma_{13}, \sigma_{14}, \sigma_{25}, \sigma_{26}\right)^{T} \mid \varsigma_{12}+\sigma_{13}+\sigma_{14} \neq 0, \varsigma_{12}+\sigma_{25}+\sigma_{26} \neq 0\right\},
$$

the canonical dual problem to the $\delta$-perturbed problem $\left(\mathcal{P}_{\delta}\right)$ is

$$
\left(\mathcal{P}_{\delta}^{d}\right): \max \left\{\Pi_{\delta}^{d}(\boldsymbol{\varsigma}, \boldsymbol{\sigma}) \mid(\boldsymbol{\varsigma}, \boldsymbol{\sigma}) \in \mathcal{S}_{a}\right\},
$$


where

$$
\begin{aligned}
\Pi_{\delta}^{d}(\boldsymbol{\varsigma}, \boldsymbol{\sigma})=- & \frac{1}{2} F_{\delta}(\boldsymbol{\sigma})^{T} \mathbf{G}^{-1}(\boldsymbol{\varsigma}, \boldsymbol{\sigma}) F_{\delta}(\boldsymbol{\sigma})-\left(d_{12}\right)^{2} \varsigma_{12}+\left(\mathbf{a}_{3}^{T} \mathbf{a}_{3}-\left(d_{13}\right)^{2}\right) \sigma_{13} \\
+ & \left(\mathbf{a}_{4}^{T} \mathbf{a}_{4}-\left(d_{14}\right)^{2}\right) \sigma_{14}+\left(\mathbf{a}_{5}^{T} \mathbf{a}_{5}-\left(d_{25}\right)^{2}\right) \sigma_{25} \\
+ & \left(\mathbf{a}_{6}^{T} \mathbf{a}_{6}-\left(d_{26}\right)^{2}\right) \sigma_{26}-\frac{1}{2} \varsigma_{12}^{2}-\frac{1}{2} \sigma_{13}^{2}-\frac{1}{2} \sigma_{14}^{2}-\frac{1}{2} \sigma_{25}^{2}-\frac{1}{2} \sigma_{26}^{2} \\
F_{\delta}(\boldsymbol{\sigma})= & \left(\delta_{1}^{1}+2 a_{3}^{1} \sigma_{13}+2 a_{4}^{1} \sigma_{14}, \delta_{1}^{2}+2 a_{3}^{2} \sigma_{13}+2 a_{4}^{2} \sigma_{14}\right. \\
& \left.\delta_{2}^{1}+2 a_{5}^{1} \sigma_{25}+2 a_{6}^{1} \sigma_{26}, \delta_{2}^{2}+2 a_{5}^{2} \sigma_{25}+2 a_{6}^{2} \sigma_{26}\right)^{T}
\end{aligned}
$$

$$
\begin{aligned}
& \mathbf{G}(\boldsymbol{\varsigma}, \boldsymbol{\sigma})= \\
& {\left[\begin{array}{cccc}
2\left(\varsigma_{12}+\sigma_{13}+\sigma_{14}\right) & 0 & -2 \varsigma_{12} & 0 \\
0 & 2\left(\varsigma_{12}+\sigma_{13}+\sigma_{14}\right) & 0 & -2 \varsigma_{12} \\
-2 \varsigma_{12} & 0 & 2\left(\varsigma_{12}+\sigma_{25}+\sigma_{26}\right) & 0 \\
0 & -2 \varsigma_{12} & 0 & 2\left(\varsigma_{12}+\sigma_{25}+\sigma_{26}\right)
\end{array}\right]}
\end{aligned}
$$

Set $\boldsymbol{\delta}=[0.005,0.005,0.005,0.005]^{T}$. Then, the canonical dual problem has a unique solution [19]

$$
\begin{aligned}
(\overline{\boldsymbol{\zeta}}, \overline{\boldsymbol{\sigma}}) & =\left[\varsigma_{12}, \sigma_{13}, \sigma_{14}, \sigma_{25}, \sigma_{26}\right]^{T} \\
& =[-0.0000,0.0005,0.0020,-0.0020,-0.0005]^{T} .
\end{aligned}
$$

By Theorem 4, it follows that

$$
\begin{aligned}
\overline{\mathbf{y}} & =\left[\bar{x}_{1}^{1}, \bar{x}_{1}^{2}, \bar{x}_{2}^{1}, \bar{x}_{2}^{2}\right]^{T}=\mathbf{G}^{-1}(\overline{\boldsymbol{\zeta}}, \overline{\boldsymbol{\sigma}}) F_{\delta}(\overline{\boldsymbol{\sigma}}) \\
& =[-0.9994,0.0002,1.0006,0.0002]^{T}
\end{aligned}
$$

is a global minimizer of $\Pi_{\delta}(\mathbf{y})$.

It is easy to verify that

$$
\Pi_{\delta}(\overline{\mathbf{y}})=-4.1667 \times 10^{-6}=\Pi_{\delta}^{d}(\overline{\boldsymbol{\zeta}}, \overline{\boldsymbol{\sigma}}) .
$$

By the fact that

$$
\Pi(\overline{\mathbf{y}})=4.1667 \times 10^{-6},
$$

the $\delta$-perturbed solutions $\overline{\mathbf{y}}$ can be considered as the global minimizer to the original problem $\left(\mathcal{P}_{0}\right)$ and we have

$$
\begin{aligned}
& \left\|\mathbf{x}_{1}-\mathbf{x}_{2}\right\|=2.0000,\left\|\mathbf{x}_{1}-\mathbf{a}_{3}\right\|=2.0001,\left\|\mathbf{x}_{1}-\mathbf{a}_{4}\right\|=2.0005 \\
& \left\|\mathbf{x}_{2}-\mathbf{a}_{5}\right\|=1.9995,\left\|\mathbf{x}_{2}-\mathbf{a}_{6}\right\|=1.9999
\end{aligned}
$$




\subsection{8 sensors network localization problem with four anchors}

We now consider sensor network localization problem with 18 sensors. In this case, we have Problem $\left(\mathcal{P}_{1}\right)$ with $d=2$. Define $\mathbf{y}=\left[x_{1}^{1}, x_{1}^{2}, \cdots, x_{n}^{1}, x_{n}^{2}\right]^{T} \in$ $\mathbb{R}^{2 n}$, and let $w_{i j}=q_{i k}=1$ in Problem $\left(\mathcal{P}_{1}\right)$. Here, we do not consider noise.

The 18 sensors $\left\{\hat{\mathbf{x}}_{i}=\left[\hat{x}_{i}^{1}, \hat{x}_{i}^{2}\right]: i=1, \cdots, 18\right\}$ are randomly generated in the unit square $[-0.5,0.5] \times[-0.5,0.5]$. The four anchors $\left(\mathbf{a}_{1}, \mathbf{a}_{2}, \mathbf{a}_{3}, \mathbf{a}_{4}\right)$ are placed at the positions $( \pm 0.45, \pm 0.45)$. The distances $\mathbf{d}=\left\{d_{i j}\right\}, i=$ $1, \cdots, 18 ; j=1, \cdots, 18$, and $\mathbf{e}=\left\{e_{i k}\right\}, i=1, \cdots, 18 ; k=1, \cdots, 4$, are computed as follows:

$$
d_{i j}=\left\|x_{i}^{*}-x_{j}^{*}\right\|, e_{i k}=\left\|x_{i}^{*}-a_{k}\right\|
$$

We now assume that the locations of the 18 sensors are unknown. They are to be determined by the approach proposed in the paper. The sequential quadratic programming approximation with active set strategy in the optimization toolbox within the Matlab environment is used to solve the canonical dual problem.

By Theorem 4, we obtain $\overline{\mathbf{y}}=\left[\overline{\mathbf{x}}_{1}, \cdots, \overline{\mathbf{x}}_{18}\right]^{T}$ with $\overline{\mathbf{x}}_{i}=\left[\overline{\mathbf{x}}_{i}^{1}, \overline{\mathbf{x}}_{i}^{2}\right]^{T}, i=$ $1, \cdots, 18$, which is a global minimizer of $\Pi(\mathbf{y})$.

Furthermore, we have

$$
\Pi(\overline{\mathbf{y}})=1.30 \times 10^{-8} \simeq 3.03 \times 10^{-8}=\Pi^{d}(\overline{\boldsymbol{\zeta}}, \overline{\boldsymbol{\sigma}}) .
$$

This problem is also solved by the standard semi-definite programming (SDP) method. The RMSD obtained using the canonical dual method is $4.61 \times 10^{-7}$, while the RMSD obtained using the standard SDP method is $4.45 \times 10^{-5}$, where RMSD is the Root Mean Square Distance defined by

$$
\operatorname{RMSD}=\left(\frac{1}{n} \sum_{i=1}^{n}\left\|\hat{\mathbf{x}}_{i}-\overline{\mathbf{x}}_{i}\right\|^{2}\right)^{\frac{1}{2}},
$$

which is to measure the accuracy of the computed locations.

The computed results by the canonical dual method and the standard SDP method are plotted in Fig. 3 and Fig. 4, respectively. The true sensor locations (denoted by circles) and the computed locations (denoted by stars) are connected by solid lines. Our program is implemented in the MATLAB environment, where SEDUMI [30] is used as the SDP solver. From the results obtained, we see that, when there is no noise and the sensor size is not too large, both the canonical dual method and SDP method are very effective 


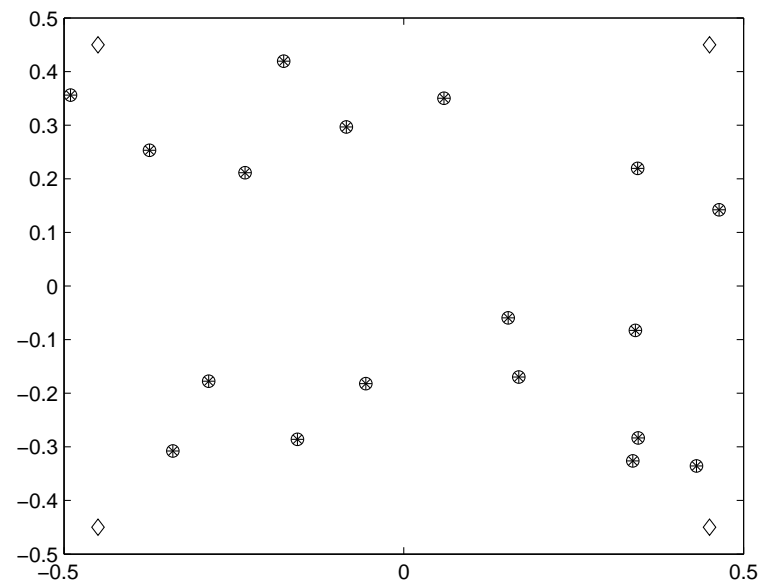

Figure 3: Sensor network with 18 sensors by the canonical dual method.

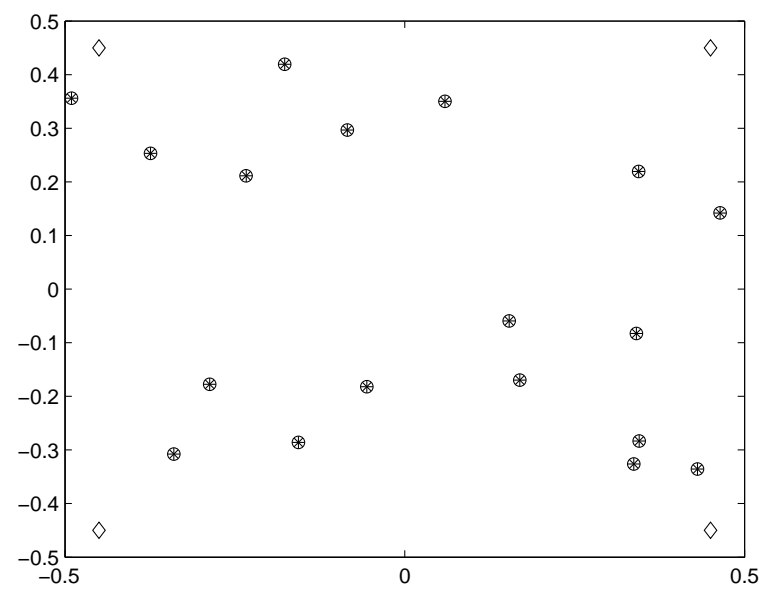

Figure 4: Sensor network with 18 sensors by the standard SDP method.

method for finding sensor locations. In particular, for the canonical dual method, all the stars are exactly located inside circles. The computational time for canonical dual method and SDP method are 0.61 seconds and 21.53 seconds, respectively. 


\subsection{A 20-sensor-network localization problem with distance errors}

A network of 20 uniform randomly distributed unknown points is generated in the square area $[0,1] \times[0,1]$. We assume:

$$
\begin{aligned}
& \text { if }\left\|x_{i}-x_{j}\right\| \leq \text { radio range, a distance (with noise) is given between } \\
& x_{i} \text { and } x_{j} \text {; } \\
& \text { if }\left\|x_{i}-x_{j}\right\|>\text { radio range, no distance is given between } x_{i} \text { and } x_{j} \text {. }
\end{aligned}
$$

Also, there are four anchors are located in $[0,0],[0,1],[1,0]$ and $[1,1]$. The distances between the nodes are calculated. If the distance between two nodes is within the specified radio range of 0.4 , the distance is included in the edge set for solving the problem after adding a random error to it in the following manner:

$$
d_{i j}=\hat{d}_{i j}|1+N(0,0.001)|
$$

where $\hat{d}_{i j}$ is the actual distance between the 2 nodes, and $N(0,0.001)$ is a random variable.

The computed results obtained by the canonical dual method and the standard SDP method [27] are plotted in Fig. 5] and Fig. 6, respectively. The true sensor locations (denoted by circles) and the computed locations (denoted by stars) are connected by solid lines. The computational time for canonical dual method and SDP method are 0.65 seconds and 27.71 seconds.

\subsection{A 200-sensor-network localization problem with distance errors}

A network of 200 uniform randomly distributed unknown points is generated in the square area $[0,1] \times[0,1]$. Four anchors are located in $[0,0],[0,1]$, $[1,0]$ and $[1,1]$. For all sensors, the radio range $=0.3$. The distance, including a random error, is generated in the following manner:

$$
d_{i j}=\hat{d}_{i j}|1+N(0,0.001)|
$$

The computed results obtained by the quadratic perturbed canonical dual method and the standard SDP method are plotted in Fig. 6 and Fig. 8, respectively. Careful examination of the results obtained for the cases involving 20 sensors and 200 sensors, we observe that when noise is taken into consideration, the canonical dual method gives rise to much better solutions. 


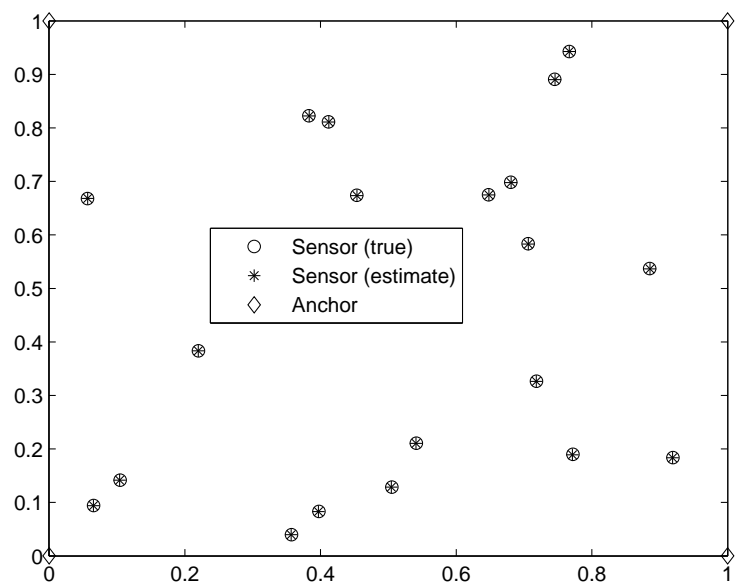

Figure 5: Sensor network with 20 sensors solved by the canonical dual method.

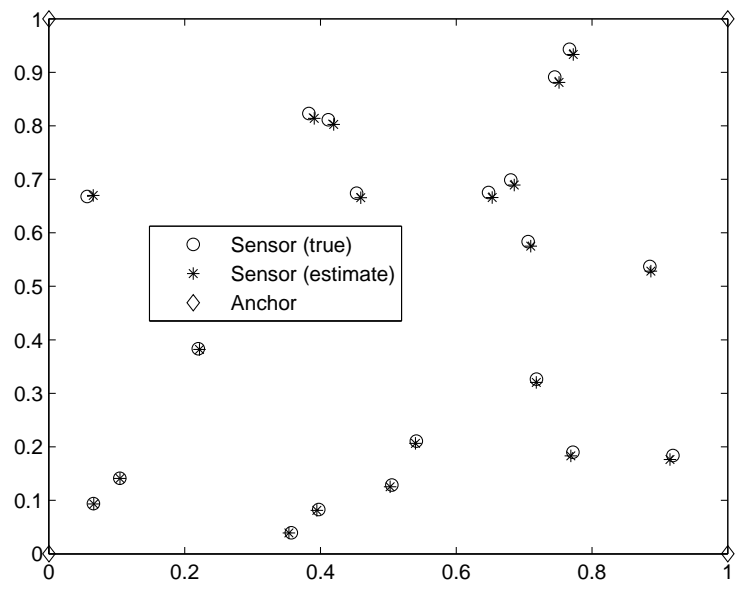

Figure 6: Sensor network with 20 sensors solved by the standard SDP method.

In particular, if the level of noise or the sensor size is large, the standard SDP is usually having difficulty to finding the exact sensor positions, see Figure 6 and Figure 8. The computational time for canonical dual method and SDP method are 127.10 seconds and 1088.70 seconds, respectively. 


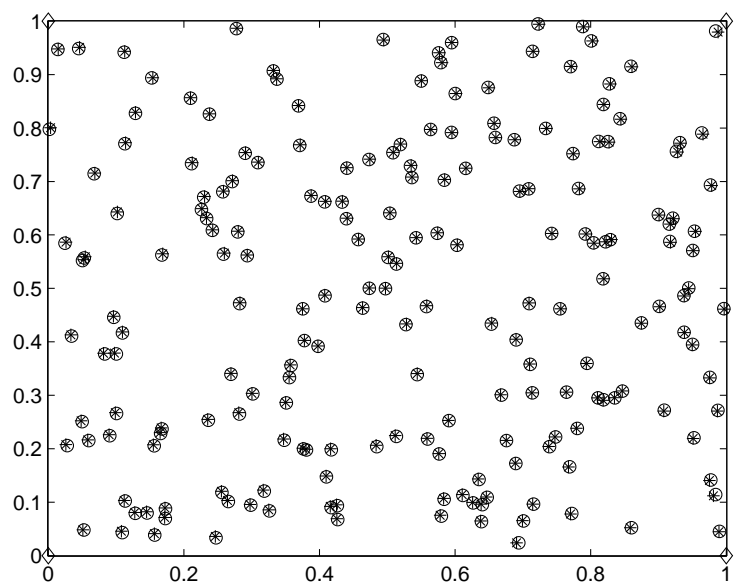

Figure 7: Sensor network with 200 sensors solved by the canonical dual method.

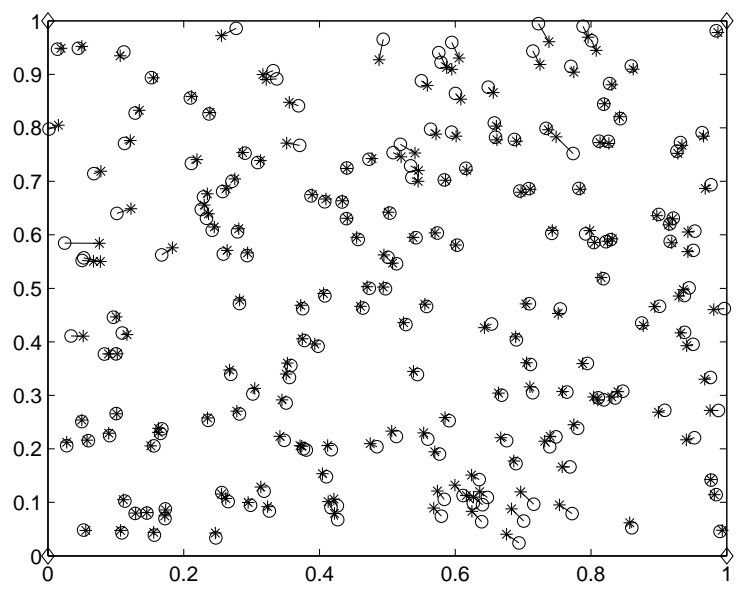

Figure 8: Sensor network with 200 sensors solved by the standard SDP method.

\section{Conclusion Remarks}

We have presented a solid application of the canonical duality theory for solving a general sensor network localization problem. By using the complementary-dual principle, a general form of analytical solution form is obtained in terms of the canonical dual variables. Based on a perturba- 
tion method, an effective canonical saddle min-max approach is proposed for solving this challenging problem. Our results show that the general sensor localization problem is not NP-hard if its canonical dual or perturbed problem has a solution in $\mathcal{S}_{a}^{+}$. Applications are illustrated by detailed analysis of a small size problem as well as some relatively large scale sensor network localization problems.

From mechanics point of view, a sensor network is similar to a structure, for the given boundary conditions (anchors) and external force $\mathbf{f}$ (linear perturbation), as long as the problem is statically determinate, i.e. the canonical equilibrium equation (44) has a solution (see page 199 [13]), the canonical dual problem has a critical point in $\mathcal{S}_{a}^{+}$and the sensor location problem can be solved efficiently regardless of its size. By the definition (18) of $\mathbf{G}(\boldsymbol{\varsigma})$ we know that $\mathcal{S}_{a}^{+} \neq \emptyset$ as long as there exists at least one $\mathbf{H}_{k l} \succ 0$ (this condition satisfied naturally for any objective measure $\Lambda(\mathbf{x}))$. The necessary condition has been studied in [17] (Theorem 8) and [19] (Section 4) for $\mathcal{S}_{a}^{+}$has a critical point of $\Pi^{d}(\boldsymbol{\varsigma})$. However, the sufficient condition is still open, which is fundamentally important for using linear perturbation method to solve NP-hard problems. Nevertheless, the quadratic perturbation methods introduced in [19] as well as in this paper (51) provide more robust approach for solving this type of challenging problems. Finally, the performance of the method proposed on problems with noisy distance data needs further investigation.

Acknowledgment: The authors are gratefully indebted with the handling editor for his detailed remarks and important suggestions. This paper has benefited greatly from two anonymous referees' comprehensive and constructive comments. The main ideas and results of this paper have been announces at the 19th International Conference on Neural Information Processing (ICONIP2012), Nov. 12-15, 2012, Doha, Qatar as well as several tutorial/plenary lectures in 2013 including the 3rd World Congress of Global Optimization, July 7-12, 2013, the Yellow Mountains, China. This work was partially supported by a grant (AFOSR FA9550-10-1-0487) from the US Air Force Office of Scientific Research. Dr. Ning Ruan was supported by a funding from the Australian Government under the Collaborative Research Networks (CRN) program. 


\section{References}

[1] A. Alfakin, Graph rigidity via euclidean distance matrices,Linear Algebra Appl., 310 (2000) 149-165.

[2] J. Aspnes, D. Goldberg and Y.R. Yang. On the computational complexity of sensor network localization. Lecture Notes in Computer Science (3121), Springer-Verlag, 2004, pp.32-44.

[3] A. Bishop, B. Fidan, B. O. Anderson, K. Dogcancay, and P. Pathirana, Optimality analysis of sensor-target localization geometries, Automatica, 46 (2010) 479-492

[4] P. Biswas, T.C. Lian, T.C. Wang and Y.Y. Ye, Semidefinite programming based algorithms for sensor network localization, ACM Trans. Sen. Netw., 2 (2006) 188-220.

[5] K. Cai, D.Y. Gao, and Q. Qin, Post-buckling solutions of hyper-elastic beam by canonical dual finite element method, Mathematics and Mechanics of Solids (2013). http://mms.sagepub.com/content/early/2013/05/06/1081286513482483.full.pdf

[6] Y. Chen and D.Y. Gao, Global solutions to large-scale spherical constrained quadratic minimization via canonical dual approach, to appear in Optimization Methods and Software, http://arxiv.org/abs/1308.4450

[7] M. Chiang, Nonconvex optimization for communication systems, in Advances in Applied Mathematics and Global Optimization D.Y Gao and H. Sherali, Eds., Berlin, Germany: Springer-Verlag, 2009.

[8] M. Deghat, I. Shames, B. Anderson, and J. Moura, Distributed localization via barycentric coordinates: Finite-timeconvergence, in 18th World Congress of the International Federation of Automatic Control,(IFAC 2011), Milan, Italy, 2011, 7824-7829.

[9] I. Ekeland and R. Temam, Convex Analysis and Variational Problems, NorthHolland (1976).

[10] S.C. Fang, D.Y. Gao, R.L. Sheu, and S.Y. Wu, Canonical dual approach for solving 0-1 quadratic programming problems, J. Ind. and Manag. Optim. 4, 125-142 (2008).

[11] D.Y. Gao, Nonlinear elastic beam theory with applications in contact problem and variational approaches, Mech. Research Commun., 23 (1) (1996), 11-17. 
[12] D.Y. Gao, Dual extremum principles in finite deformation theory with applications to post-buckling analysis of extended nonlinear beam theory, Appl. Mech. Rev., 50 (11) (1997), S64-S71.

[13] D.Y. Gao, Duality Principles in Nonconvex Systems: Theory, Methods and Applications, Dordrecht/Boston/London: Kluwer Academic Publishers, 2000 .

[14] D.Y. Gao, Perfect duality theory and complete solutions to a class of global optimization problems, Optimisation, 52 (4-5)(2003), 467-493.

[15] D.Y. Gao, Nonconvex semi-linear problems and canonical duality solutions, Advances in Mechanics and Mathematics, Kluwer Academic Publishers, Dordrecht/Boston/London, Vol. II (2003), 261-312.

[16] D.Y. Gao, Solutions and optimality to box constrained nonconvex minimization problems, J. Ind. Manag. Optim., 3(2) (2007) 293-304.

[17] D.Y. Gao, Canonical duality theory: theory, method, and applications in global optimization. Comput. Chem. 33, 1964-1972, (2009).

[18] D.Y. Gao and R.W. Ogden, Multiple solutions to non-convex variational problems with implications for phase transitions and numerical computation. Q. J. Mech. Appl. Math, Vol. 61. No. 4 (2008) 497-522.

[19] D.Y. Gao and N. Ruan, Solutions to quadratic minimization problems with box and integer constraints, J. Global Optim., 47(2010) 463-484.

[20] D.Y. Gao, N. Ruan, and P. Pardalos, Canonical dual solutions to sum of fourth-order polynomials minimization problems with applications to sensor network localization, in Sensors: Theory, Algorithms, and Applications, V. Boginski, C. W. Commander, P. Pardalos, and Y. Ye, Eds., New York: Springer, 61 (2012) 37-54.

[21] D.Y. Gao and H.D. Sherali, Canonical duality: Connection between nonconvex mechanics and global optimization, in Advances in Appl. Mathematics and Global Optimization, 249-316, Springer (2009).

[22] D.Y. Gao and G. Strang, Geometric nonlinearity: Potential energy, complementary energy, and the gap function. Quart. Appl. Math. 47(3), 487-504 (1989).

[23] D.Y. Gao and C. Wu, On the triality theory for a quartic polynomial optimization problem, J. Ind. Manag. Optim., 8(1) (2012) 229-242. 
[24] D.Y. Gao and H. Yu, Multi-scale modelling and canonical dual finite element method in phase transitions of solids. Int. J. Solids Struct., 45 (2008) 36603673 .

[25] M. Huang, S. Chen, and Y. Wang, Minimum cost localization problem in wireless sensor networks, Ad Hoc Netw., 9 (2011) 387-399.

[26] U. A. Khan, S. Kar, and J. Moura, Distributed sensor localization in random environments using minimal number of anchor nodes, IEEE Trans. Signal Process., 57(5) (2009) 2000-2016.

[27] S. Kim, M. Kojima, and H. Waki, Exploiting sparsity in SDP relaxation for sensor network localization, SIAM J. Optimiz., 20 (2009) 192-215.

[28] V. Latorre and D.Y. Gao, Canonical duality for RBF neural networks, to appear in Neuralcomputings (2013).

[29] S.F. Li and A. Gupta, On dual configuration forces, J. of Elasticity, 84:13-31 (2006).

[30] W. Lu, Use SeDuMi to solve LP, SDP and SCOP problem: Remarks and examples. [Online]. Available: http://www.ece.uvic.ca/ wslu/Talk/SeDuMi-Remarks.pdf

[31] D. Morales and D.Y. Gao, On the minimal distance between two surfaces, to appear in Optimization, Published online at http://arxiv.org/abs/1210.1618

[32] J. More and Z. Wu. Global continuation for distance geometry problems, SIAM Journal on Optimization, 7(1997), 814-836.

[33] A. Pal, Localization algorithms in wireless sensor networks:current approaches and future challenges, in Network Protocols and Algorithms, 2(1) (2010) 45-74.

[34] T. Pong and P. Tseng, (robust) edge-based semidefinite programming relaxation of sensor network localization, Math. Program. (ser. A), 130 (2011) 321-358.

[35] R. Rangarajan, R. Raich, and A. Hero, Euclidean matrix completion problems in tracking and geo-localization, in IEEE Intl Conf. on Acoustics, Speech and Signal Processing, Apple, 2008. 
[36] N. Ruan and D.Y. Gao, Canonical duality approach for nonlinear dynamical systems, IMA J. Appl. Math (2013). Published online http://arxiv.org/abs/1206.2447

[37] N. Ruan, D. Y. Gao, and Y. Jiao, Canonical dual least square method for solving general nonlinear systems of quadratic equations, Comput. Optim. Appl., 47 (2008) 335-347.

[38] H. Santosa and D.Y. Gao, Canonical dual finite element method for solving post-buckling problems of a large deformation elastic beam, Int. J. Nonlinear Mechanics, 47 (2012), 240-247, doi:10.1016/j.ijnonlinmec.2011.05.012

[39] D. Morales-Silva and D.Y. Gao, On the minimal distance between two surfaces, to appear in Optimization. http://arxiv.org/abs/1210.1618

[40] W. Sun and Y. X. Yuan,Optimization Theory and Methods: Nonlinear Programming, New York: Springer, 2006.

[41] P. Tseng, Second-order cone programming relaxation of sensor network localization, SIAM J. Optimiz., 18(1) (2007) 156-185.

[42] Z.B. Wang, S.C. Fang, D.Y. Gao, and W.X. Xing, Canonical dual approach to solving the maximum cut problem, J. Glob. Optim., 54 (2012), 341351. DOI 10.1007/s10898-012-9881-8

[43] Z.Wang, S. Zheng, Y. Ye, and S. Boyd, Further relaxation of the semidefinite programming approach to sensor network localization, SIAM J. Optimiz., 19(2) (2008) 655-673.

[44] J. Zhang, D. Gao, and J. Yearwood, A novel canonical dual computational approach for prion agaaaaga amyloid fibril molecular modeling, J. Theoretical Biology, 284 (2011) 149-157.

[45] P. Zhang and M. Martonosi, Locate: Collabotive localization estimation for sparse mobile sensor networks, in The International Conference on Information Processing in Sensor Networks, Milan, Italy, Apple 2008, (IPSN 2008). 